\title{
A mini review of paragangliomas with presentation of two cases
}

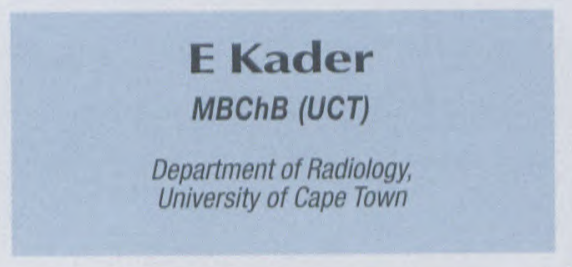

\section{Abstract}

Paragangliomas are rare tumours of the

paraganglion system, which may, in approximately $80 \%$ of cases, be hormonally active with potentially lethal consequences. In this mini review, the classification, pathology, pathophysiology and clinical management are described. An attempt is also made to evaluate the relative advantages and disadvantages of the most commonly used diagnostic imaging modalities, viz. Magnetic Resonance Imaging (MRI), Computed Tomography (CT) and radionuclide scintigraphy.

Paragangliomas are divided into four anatomical classes:
1. Paragangliomas arising from the adrenal medulla

2. Aortico-sympathetic paragangliomas

3. Parasympathetic and visceroautonomic paragangliomas 4. Paragangliomas not otherwise specified. They occur sporadically or in families, and have recognised associations with other diseases. The majority are benign.

Ninety percent of tumours are adrenal and $10 \%$ are extra-adrenal. Patients commonly present with

hypertension or paroxysmal crises and the laboratory diagnosis hinges on the demonstration of elevated catecholamine levels. Initial management involves stabilisation with alphablockade followed by preoperative tumour localisation. MRI has similar accuracy to CT in diagnosis of adrenal tumours. MRI surpasses metaiodobenzylguanidine (MIBG) scanning in detecting adrenal disease and nonfunctional paragangliomas. MIBG has greater sensitivity and specificity than MRI in the detection of extraadrenal tumours, although both are superior to CT. MRI is currently the investigation of choice, with MIBG being used in cases of disease recurrence, metastases or equivocal MRI.

Surgical cure is possible in up to $85 \%$ of cases. The recurrence rate is between 5 and $10 \%$, with a 5 year survival rate of 80 to $95 \%$.

\section{Introduction}

Paragangliomas are rare tumours arising from the paraganglion system. The incidence in the general population is $0.001-0.002 \%$, and 0.1 to $0.5 \%$ in those with hypertension. ${ }^{1,2}$ Affected individuals may be asymptomatic if the tumour is non-functional, or can present with a broad spectrum of symptoms in the case of functional tumours. These tumours carry a high morbidity and mortality if discovered incidentally. Diagnosis relies on a high clinical index of suspicion and appropriate biochemical evaluation. Subsequent management includes stabilisation of the patient and preoperative evaluation prior to surgical excision. Surgical cure rates of $66-85 \%$ have been reported, given early intervention. ${ }^{2,3}$ 


\section{A mini review of paragangliomas with presentation of two cases}

\section{from page 11}

This review attempts to address the recommended nomenclature and classification of these tumours, as well as their basic pathological and pathophysiological features. Numerous biochemical tests are currently employed in diagnosis, and their relative merits and demerits are compared. MRI, CT and nuclear medicine (NM) scanning have largely replaced more invasive techniques in preoperative tumour localisation. The relative advantages, disadvantages and recommended application of these three modalities are considered.

\section{Case 1}

A 20-year-old man presented with a brief history of lower abdominal pain accompanied by nausea and vomiting. He had been entirely well prior to this presentation and had no significant family history. On examination the patient was apyrexial, normotensive and not tachycardic. Tenderness was noted in the left lower abdominal quadrant. The chest radiograph was normal. The abdominal $\mathrm{x}$-ray revealed a soft tissue density to the left of the umbilicus. This was confirmed on ultrasound. Catecholamine assays were normal. The CT scan demonstrated a $5 \mathrm{~cm}$ lesion with a central low density at the aortic bifurcation (Figure 1). On the basis of the inconclusive findings above, an $I^{123}$ metaiodobenzylg u a n i d in e (MIBG) scan was performed and revealed an area of increased uptake in the region of the aortic bifurcation (Figure 2).

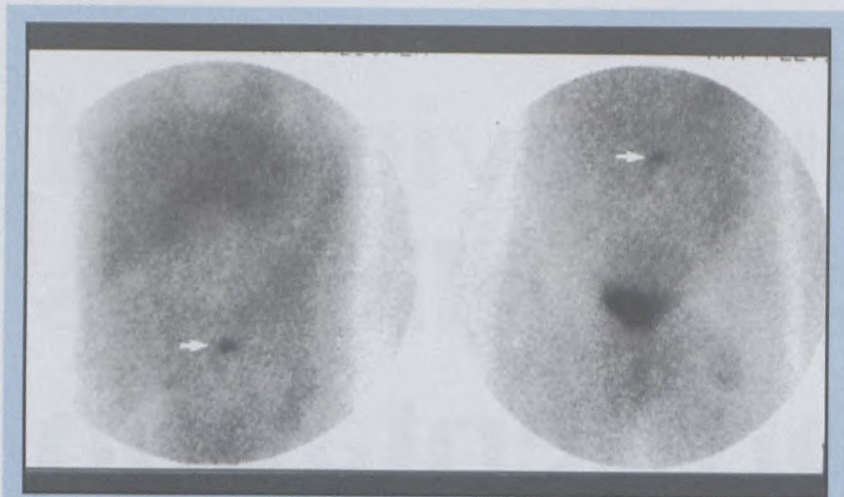

Figure 2: MIBG scan showing an area of increased uptake in the region of the aortic bifurcation (arrows).

The lesion was excised and was histologically confirmed to be a paraganglioma.

\section{Case 2}

A 19-year-old man presented with a history of progressive dyspnoea, loss of weight and palpitations. He was admitted to hospital in pulmonary oedema secondary to malignant hypertension. He had been investigated a year earlier for lethargy, episodic headache, palpitations and epigastric pain with nausea and vomiting. On examination he was tachycardic and hypertensive (blood pressure was 183/139 $\mathrm{mmHg}$ ) with clinical evidence of cardiac failure. Fundoscopy revealed grade 3 hypertensive retinopathy. A chest

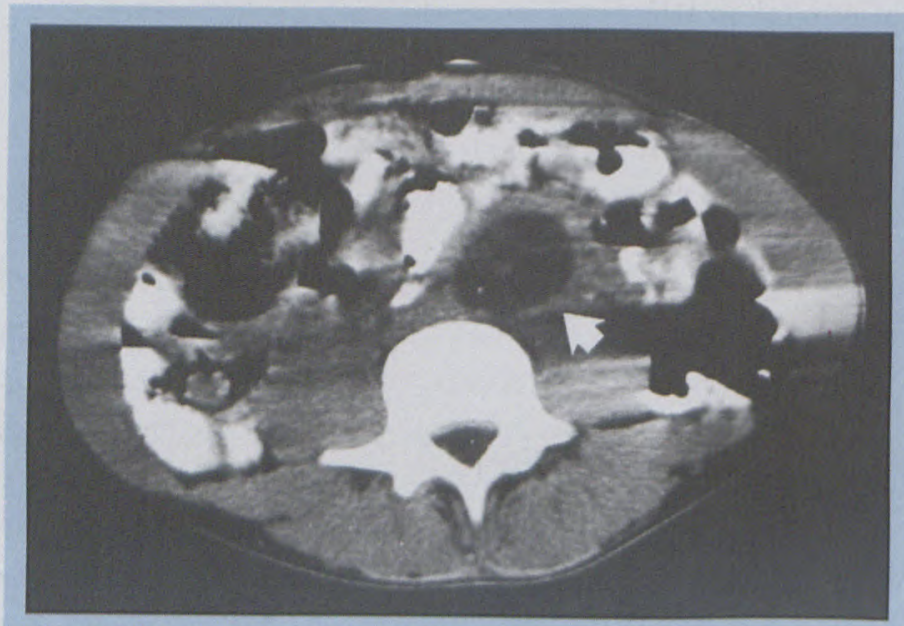

Figure 1: Computed tomogram with intravenous contrast medium demonstrating a rounded soft-tissue mass with central low density (arrow) anterior to the lumbar spine. radiograph confirmed pulmonary oedema. The abdominal x-ray was normal. The plasma noradrenaline level was 16500 (normal range 80-2 200) and urine NMA was 90.9 (normal range 0-5).A large, para-aortic soft tissue mass in the left infrarenal area was noted on ultrasound (US) (Figure 3) and CT (Figure 4). The mass was surgically excised and histology confirmed a paraganglioma.
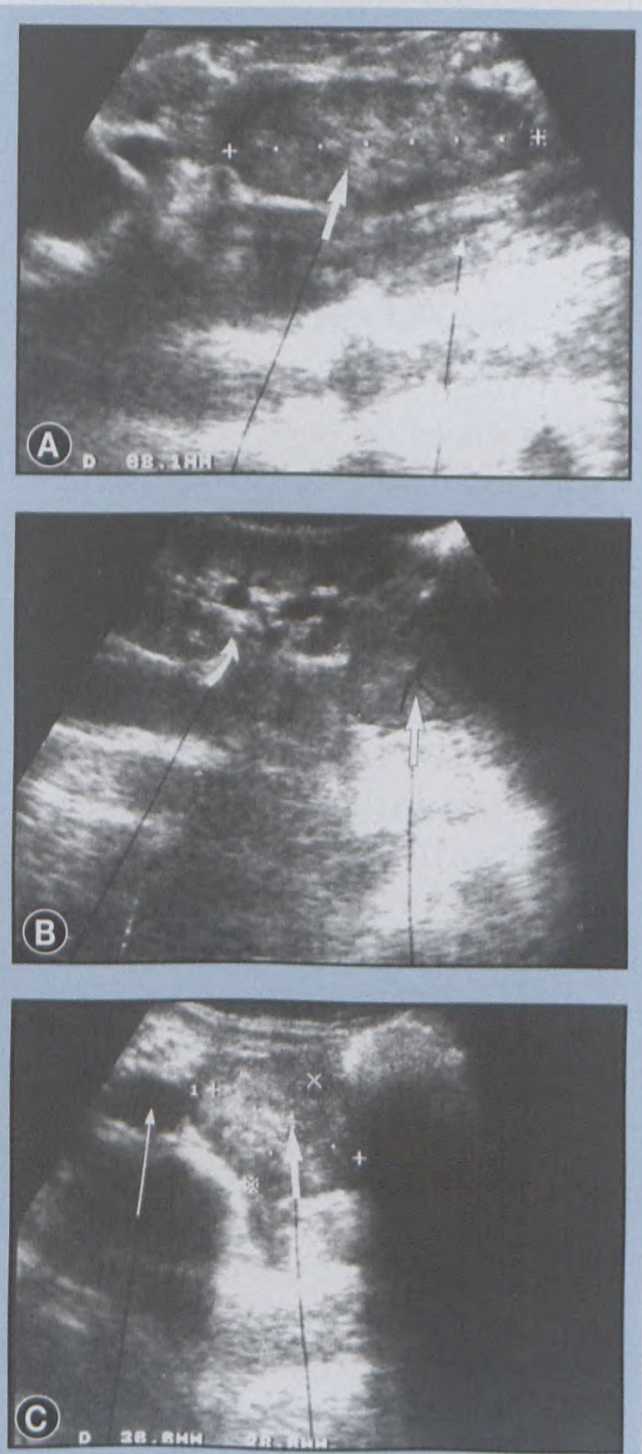

Figure 3a-c: Longitudinal ( $3 a$ and $b$ ) and transverse ( $3 c$ ) ultrasound scans demonstrating an infra-renal 6.8 by $2.9 \mathrm{~cm}$ inhomogeneous lesion in the left para-aortic area. The relationship of the lesion (thick arrows) to the left kidney (curved arrows), aorta (thin arrows), and psoas muscle (arrowhead) can be appreciated. 
from page 12

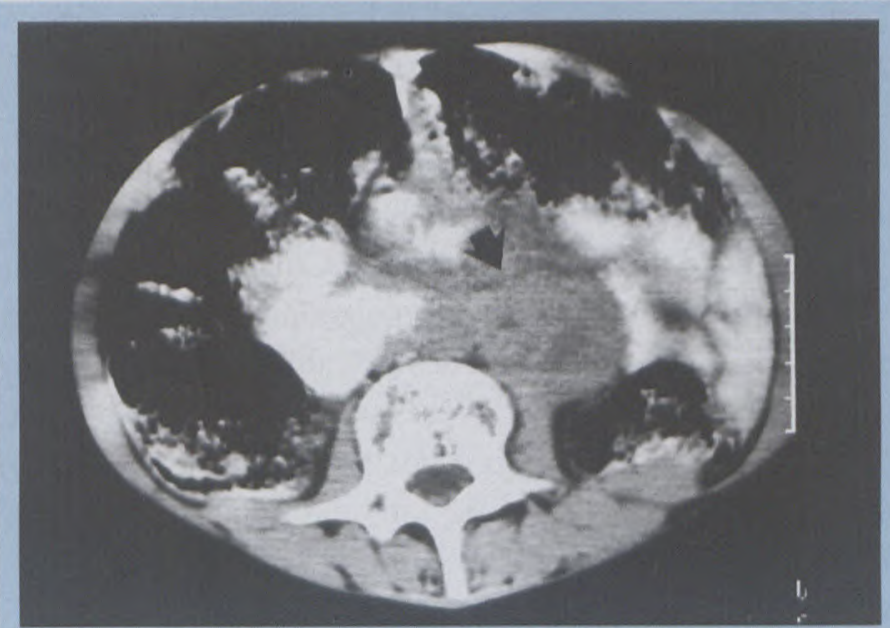

Figure 4: Computed tomogram (without intravenous contrast agent) of the lower abdomen demonstrating an inhomogeneous, soft-tissue mass in the left para-aortic region (arrow).

\section{Anatomy}

The paraganglion system is part of the Amine Precursor Uptake and Decarboxylation (APUD) system (or "diffuse neuroendocrine system") which modulates the function of the nervous and endocrine systems. Paraganglion cells are derived from neuroectoderm and are topographically and physiologically integrated with the autonomic nervous system. 1,3,4 Aggregations of cells associated with the sympathetic nervous system (chromaffin cells) constitute the chromaffin system and can conveniently be conceptualised as being adrenal medullary or extra-adrenal. The extra-adrenal cell groups are called paraganglia and occur in or near the sympathetic chain, near major blood vessels, or in various organs. In addition, paraganglion cells are also encountered in association with parasympathetic tissue..$^{1-4}$

Glenner and Grimley, in 1974, classified paraganglia, and tumours arising from them, into four classes, viz. branchiomeric, intravagal, aorticosympathetic, and visceroautonomic. ${ }^{5}$ Tumours arising from the first two classes of paraganglia usually occur in the head and neck and generally stain negative for chromaffin, while tumours of the latter two groups occur along the aorta, in association with blood vessels or visceral organs, and are usually chromaffin positive. This classification is very similar to the more recent WHO classification of 1980, which defines four classes of paraganglia on an anatomical basis: ${ }^{1}$ 1. Paragangliomas arising from the adrenal medulla. Only these are referred to as phaeochromocytomas.

2. Aortico-sympathetic paragangliomas associated with the sympathetic chain.

3. Parasympathetic paragangliomas (including chemodectomas) and visceroautonomic paragangliomas (found in association with small blood vessels in organs).

4.Paragangliomas not otherwise specified.

\section{Pathophysiology}

All paraganglion cells store catecholamines and may therefore be hormonally active. The hormones are not released secondary to neurological impulses, as the tumours are not innervated. Ninety percent of adrenal tumours are active, while approximately $50 \%$ of extra-adrenal tumours and $1 \%$ of parasympathetic tumours are hormonally active. ${ }^{6}$

Most paragangliomas secrete noradrenaline and/or adrenaline. Adrenal types usually secrete noradrenaline and adrenaline in ratios greater than is physiologically normal, while extraadrenal tumours tend to excrete noradrenaline exclusively. Isolated adrenaline secretion is rare. A wide variety of secreted peptides have been documented including endogenous opioids, neuropeptide $Y$, chromogranin A, dopamine, somatostatin and calcitonin. Their functional significance is dubious. ${ }^{2,7}$

\section{Pathology}

The incidence of paragangliomas in the general population is 0.001 $0.002 \%$ and $0.1-0.5 \%$ in the hypertensive population. ${ }^{1-3}$ The commonly affected age group is 25-35 years with a median age of 37-40 years. ${ }^{5,8}$ There is a known familial predisposition in $10 \%$ of cases. In these situations the incidence of multiplicity is $35 \%$, while bilateral phaeochromocytomas may occur in up to $50 \%$ of individuals, compared to $10 \%$ in sporadic cases. ${ }^{1-3,5,9}$ There is a well recognised association with other diseases, notably multiple endocrine neoplasia type $2 \mathrm{a} \& \mathrm{~b}$ (MEN 2a \& 2b), Von Hippel-Lindau disease, neurofibromatosis, Cushing's disease and Carney's triad (extra-adrenal paraganglioma, gastric leiomyosarcoma and pulmonary chondroma).

Eighty to ninety percent of paragangliomas are adrenal and 10-20\% are extra-adrenal. In the extra-adrenal group, $80 \%$ (8-16\% of all paragangliomas) are abdominal, occurring most commonly in the organ of Zuckerkandl (para-aortic body) and renal hila. One to two percent occur in the thorax, usually the posterior mediastinum. Less than $1 \%$ are found in the head and neck, commonly in the glomus caroticum (carotid body) 


\section{from page 13}

and glomus jugulare, followed by the glomus vagale and glomus tympanicum. The larynx and orbits may be involved. Approximately $1 \%$ occur in the bladder. The filum terminale is the commonest site of CNS involvement. 1,2,10

Paragangliomas are typically soft, rounded vascular tumours, less than $5 \mathrm{~cm}$ in size when extra-adrenal and less then $10 \mathrm{~cm}$ when adrenal. They are usually solid with areas of necrosis, haemorrhage or cystic change. On microscopy polyhedral cells are arranged in clusters ("zelballen") within a fibrovascular stoma. The immunohistochemical classification into chromaffin and non-chromaffin tumours is based on their reaction with chromium salts ${ }^{2,11}$ on histological staining.

Paragangliomas are usually benign and reported malignancy rates vary only slightly. Adrenal tumours are malignant in less than $10 \%$ of cases, while extra-adrenal types have a higher malignancy rate: $40 \%$ of paraaortic body tumours and $18 \%$ of vagal body tumours have metastases at presentation. It must be noted that the histological criteria for malignancy are unreliable and no significant correlation has been demonstrated between malignancy, functional status, location and histology. This diagnosis is based on local invasion and the presence of distant metastases (commonly to bone, lung, liver and lymph nodes). $1,2,8,9,11-13$

\section{Clinical manifestations}

Cervical paragangliomas present predominantly with local effects, whereas thoraco-abdominal tumours tend to present by means of humoral symptoms. The single commonest presenting symptom is recalcitrant hypertension which may be paroxysmal or constant. Less than $5 \%$ of patients present with hypotension due to excessive adrenaline release. $\mathrm{Pa}$ tients may also present with characteristic paroxysms or crises, consisting of one or more of a variety of symptoms including headaches, palpitations, tachycardia, apprehension, nausea and vomiting, syncope and chest pain. Additional features include a raised metabolic rate, carbohydrate intolerance and, in a small group of patients, adverse drug reactions, particularly to tricyclic antidepressants, opioids and monoamine oxidase inhibitors. $2,7,8,10$

\section{Diagnosis}

The diagnosis of paragangliomas is based on the demonstration of raised catecholamine levels. Radiology only has a place in the evaluation of established cases. Several laboratory tests exist, of which a few are commonly used in clinical practice. Twenty- four hour urinary catecholamine assays remain the cornerstone of diagnosis, with a sensitivity of over $96 \%$ provided specific collection criteria are adhered to. In particular, no recent radiographic contrast media should have been administered to the patient. Serum catecholamine levels are generally regarded as unreliable in isolation, because of their tendency to be normal between crises. Urine assays, in contrast, provide an integrated catecholamine value as a function of time and are less likely to render a false negative result. A further difficulty with serum assays lies in the definition of a reliable normal range. The stress of specimen collection may raise basal catacholamine levels. During anxiety or seizure attacks plasma catecholamine levels may also be elevated. In general, therefore, plasma assays are reserved for cases with equivocal urine results. Less commonly performed tests include platelet catecholamine levels, plasma chromogranin A and pharmacological tests (phentolamine based adrenolysis assessment, tyramine or glucagon induced pressor reactions, or clonidine suppression tests).

The differential diagnosis includes drug-related hypertensive crises, intracranial lesions (posterior fossa lesions, subarachnoid haemorrhage) and diencephalic epilepsy (a rare condition that presents with aura, an abnormal EEG and favourable response to anti-convulsants). ${ }^{2,3}$

\section{Management}

In the acute stage the management is resuscitative and based on control of hypertension and arrhythmias. Most paragangliomas are benign and complete surgical excision is usually possible. The preoperative management principles are:

- Induction of stable alpha-blockade, usually with phenoxybenzamine. This decreases the risk of pressor reactions and facilitates expansion of intravascular volume

- Beta-blockade for tachycardia, only after stable alpha-blockade has been achieved since unopposed betablockade may precipitate a crisis by antagonising beta-modulated peripheral vasodilation

- Metyrosine (a tyrosine hydroxylase inhibitor) may be used to inhibit catecholamine production

- Tumour localisation

Currently, tumour localisation is dominated by non-invasive imaging modalities, with infrequent use of invasive procedures. The main 


\section{from page 14}

modalities include, in the former group, MRI, nuclear medicine and CT, while arteriography and venous sampling are included in the latter. $\mathrm{Nu}$ merous studies exist comparing the relative advantages and disadvantages of the various modalities and an attempt is made to provide an overview of the prevailing opinions.

CT can detect lesions as small as $0.5 \mathrm{~cm}$ with $75-87 \%$ sensitivity. However, it may miss small adrenal lesions. Furthermore, in extra-adrenal paragangliomas, whole body scanning delivers a high radiation dose to the patient and is time consuming. In addition, intravenous iodinated contrast may precipitate a hypertensive reaction. ${ }^{14}$ On CT scanning, $55 \%$ of tumours are homogeneous, $40 \%$ have a central low density and $15 \%$ demonstrate punctate calcification. The tumours are very vascular and enhance intensely with contrast administration. ${ }^{1,8,13,15}$

MR imaging has been used for many years and in larger centres has superseded CT completely. It has been proven to be comparable in sensitivity to CT for adrenal lesions and superior to it for extra-adrenal disease. On MRI these tumours are T2 hyperintense relative to the liver. Several studies have confirmed the ability to differentiate adrenal paragangliomas from adenomas based on the tumour to liver signal ratio. Ratios greater than 2.5 suggest paraganglioma and less than 2, adenoma. ${ }^{1,16}$

Radionuclide studies are a particularly valuable tool in the preoperative assessment of the patient with paraganglioma. The two commonly used tests are MIGB (labelled with $\mathrm{I}^{123}$ or ${ }^{131}$ ) and DTPA-octreotide (labelled with $\operatorname{In}^{111}$ ). MIBG is an analogue of guanethedine and follows the same metabolic/synthetic pathway as noradrenaline, resulting in concentration within the tumour by amine uptake, but is not metabolised to any significant extent (Figure 5).

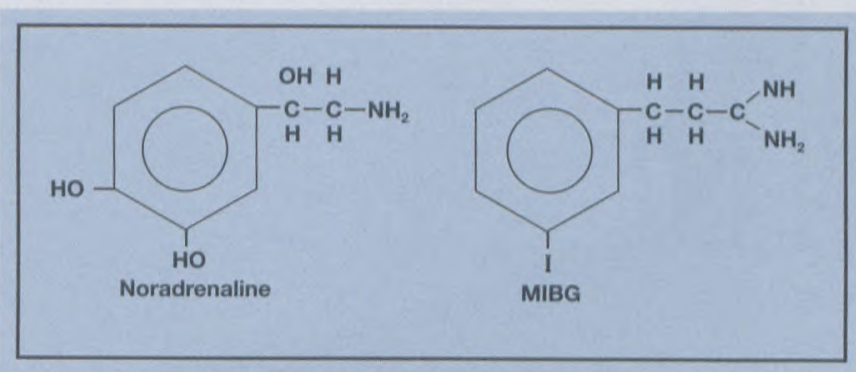

Figure 5: Molecular structure of MIBG and noradrenaline.

$\mathrm{I}^{123}$ is preferred to $\mathrm{I}^{131}$ because of the former's lack of beta emission, resulting in lower radiation dose to the patient and superior images. Many drugs can interfere with MIBG uptake including cocaine, reserpine and tricyclic antidepressants.

MRI and MIBG have comparable sensitivity for functioning tumours, but MRI is able to demonstrate non functioning tumours missed by scintigraphy. MIBG and MRI have sensitivities ranging from $83-93 \%$ and are superior to CT with sensitivities of $75-87 \%$. The specificity of MIBG is $100 \%$ and is superior to CT or MRI., ${ }^{1,13,15}$ In a study by Van Gils et al, MRI was compared to MIBG scanning, finding relative sensitivities of $91 \%$ and $80 \%$ respectively. MRI performed better in the detection of adrenal lesions ( $100 \%$ vs $75 \%$ ), while MIBG was superior in the detection of extra-adrenal disease and recurrence ( 88 vs $75 \%$ ). The overall specificities were $100 \%$ for MIBG and $82 \%$ for MRI.

The currently accepted ideal approach therefore is that MRI be used as the initial investigation and MIBG is reserved for cases of equivocal MRI results, recurrent disease, suspected metastases, multicentricity, prior to
${ }^{131}$ MIBG therapy, or where the diagnosis is in doubt (familial syndromes may have paragangliomas with normal catecholamine levels). In the case of non-functioning tumours, MRI is an acceptable alternative. Some authors advocate routine $\mathrm{MIBG}$ scanning to exclude multicentric disease, but no data exist to support this.

In 111 DTPAoctreotide is a new agent that is a somatostatin analogue which binds to receptors on neuroendocrine cells. Studies have shown sensitivities as high as $94 \%$ and superior performance when compared to MIBG. $5,9,10,17$ Its routine use is limited by lack of long-term experience and cost.

Angiography and venous sampling have a limited role in thoraco-abdominal disease. Their use is rather indicated in equivocal cases when attempting to define suspicious areas for re-investigation by axial scanning methods.

\section{Definitive treatment}

In adequately experienced surgi$\mathrm{cal}$ and anaesthetic hands operative mortality is less than $2 \% .^{2}$ Irresectable or malignant tumours, however, require medical therapy with adrenergic blockers and metyrosine. Although they are relatively radio-resistant, malignant paragangliomas show a partial response to chemotherapeutic agents including streptozocin or combined regimens such cyclophosphamide, vincristine and dacarbazine. ${ }^{3}$ 